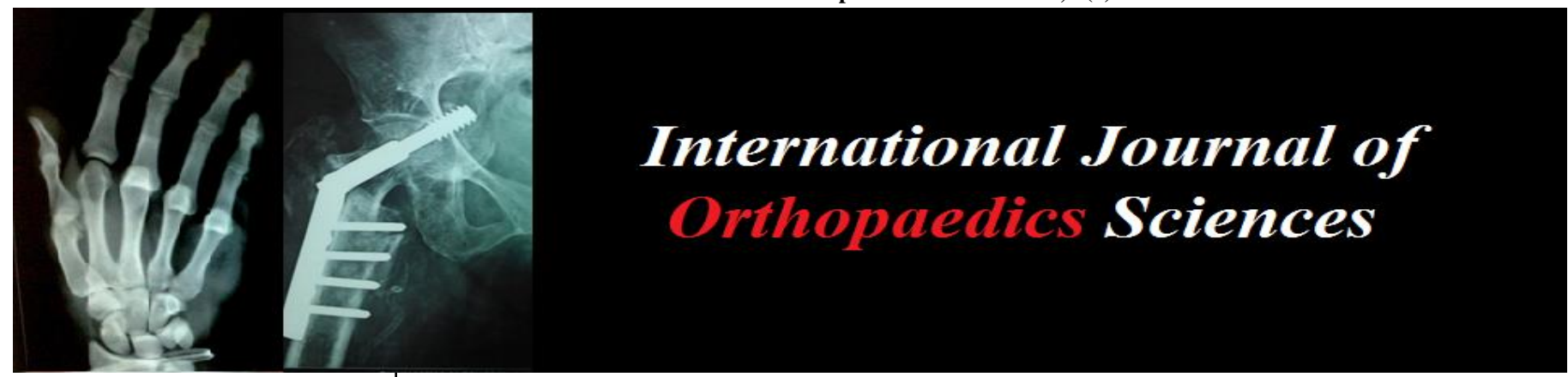

ISSN: $2395-1958$

IJOS 2019; 5(3): 508-511

(C) 2019 IJOS

www.orthopaper.com

Received: 11-05-2019

Accepted: 13-06-2019

Dr. Ankush Goyal

Assistant Professor,

Department of Orthopedics,

Adesh Institute of Medical

Sciences and Research,

Bathinda, Punjab, India

Dr. Abhishek Bansal

Assistant Professor,

Department of Orthopedics,

Adesh Institute of Medical

Sciences and Research,

Bathinda, Punjab, India
Correspondence

Dr. Abhishek Bansal

Assistant Professor,

Department of Orthopedics,

Adesh Institute of Medical

Sciences and Research,

Bathinda, Punjab, India

\section{Analysis of treatment outcome of patients with Inter- trochanteric femoral fractures undergoing surgical management by Proximal Femoral nail Antirotation (PFNA): An observational study}

\author{
Dr. Ankush Goyal and Dr. Abhishek Bansal
}

DOI: https://doi.org/10.22271/ortho.2019.v5.i3i.1579

Abstract

Background: Around 50\% of the hip fractures in the elderly are intertrochanteric fractures and half of which are unstable. The proximal femoral nail antirotation (PFNA) was designed with its novation being the helical neck blade. Hence; the present research was undertaken for assessing the outcome patients with Inter-trochanteric femoral fractures undergoing surgical management by Proximal Femoral Nail Antirotation (PFNA).

Materials \& Methods: 10 patients with Intertrochanteric femoral fractures attending the Out Patient Department/Emergency of the Department of Orthopaedics. Complete preoperative clinical examination of all the patients was carried out. All the patients underwent treatment with PFNA under the hands of skilled and experienced orthopaedic surgeon. All the patients underwent postoperative follow-up examination at subsequent visits. Both clinical and radiologic examination was done in all the patients during the postoperative period. Harris hip score was used for assessing the outcome. The statistical data is carried out using Statistical package for social sciences.

Results: Mean hospital stay was found to be 9.6 days. 40 percent of the patients had hospital stay of less than or equal to 10 days. 3 patients each (30 percent of the patients each) had mean time to union of 12 weeks and 13 weeks respectively. Overall, mean time to union was found to be 12.45 weeks. Mean Harris hip score among patients on follow-up was found to be 92.5 . In 80 percent of the patients, mean Harris hip score was found to be 91 to 100 .

Conclusion: PFNA is accompanied by early recovery and appears to be an excellent mode of surgical therapy in patients with inter-trochanteric fractures.

Keywords: Fractures, inter-trochanteric, proximal femoral nail antirotation

\section{Introduction}

Around $50 \%$ of the hip fractures in the elderly are intertrochanteric fractures and half of which are unstable. With increasing age and low bone mineral density unstable fractures are common. Intertrochanteric femur fractures were mostly subjected to conservative treatment in the past which led to a number of morbidities like external rotation deformity, malunion with varus, short limb gait and a substantial rate of mortality due to problems of recumbence and delayed mobilization, and DVT. The internal fixation of intertrochanteric fractures became popular for early restoration of the patient to their pre-injury status by increasing patient comfort, facilitating nursing care, decreasing hospitalization and reducing complications of prolonged recumbency ${ }^{[1-3]}$ The proximal femoral nail antirotation (PFNA) was designed by Synthes in 2004 with its novation being the helical neck blade. Changing the column screw into a helical blade increases the contact surface area between the purchase holding device and the femoral head cancellous bone ${ }^{[4]}$. During insertion of the blade, it compresses rather than removes the limited amount of osteoporotic bone in the proximal femur. This mechanism prevents bone loss and offers improved purchase in the femoral head due to compaction of cancellous bone around the blade during insertion. Rotation of the head-blade combination as a whole is prevented by an intrinsic locking mechanism. Furthermore, thanks to controlled impaction of the metaphyseal fracture zone, immediate full weight bearing is allowed ${ }^{[5-7]}$ 
Hence; the present research was undertaken for assessing the outcome patients with Inter-trochanteric femoral fractures undergoing surgical management by Proximal Femoral Nail Antirotation (PFNA).

\section{Materials \& Methods}

Sample size: 10 patients with Intertrochanteric femoral fractures attending the Out Patient Department/Emergency of the Department of Orthopaedics.

Ethical clearance: Obtained from ethical committee of the institution.

\section{Inclusion Criteria}

- Patients more than 50 years of age

- Patients with stable and unstable Intertrochanteric fractures

- Patients who gave informed consent for the study

\section{Exclusion Criteria}

- Patients less than 50 years of age,

- Diabetic patients,

- Hypertensive patients,

- Patients with pathologic fractures

A self-framed questionnaire was made for obtaining the demographic detail of all the patients. Complete preoperative clinical examination of all the patients was carried out. All the patients underwent treatment with PFNA under the hands of skilled and experienced orthopaedic surgeon.

Follow-up: All the patients underwent postoperative followup examination at subsequent visits. Both clinical and radiologic examination was done in all the patients during the postoperative period. Harris hip score was used for assessing the outcome ${ }^{[7]}$. The Harris hip score has a maximum of 100 points (best possible outcome) covering pain (1 item, 0-44 points), function ( 7 items, $0-47$ points), absence of deformity (1 item, 4 points), and range of motion ( 2 items, 5 points). Gradations of the total score adjudge the outcome as excellent (90-100), good (80-90), fair $(70-80)$ or poor $(<70)$.

Statistical Analysis: The statistical data is carried out using Statistical package for social sciences. Chi- square test and Mann Whitney U test were used for assessment of level of significance. P- value of less than 0.05 was taken as significant.

\section{Results}

In the present study, we analysed a total of 10 patients with inter-trochanteric femoral fractures who underwent treatment with PFNA. 60 percent of the patients of the present study were less than 60 years of age while 20 percent of the patients belonged to 71 to 80 years of age. Mean age of the patients of the present study was 58.8 years. There were 7 males (70 percent of the patients) and 3 females (30 percent of the patients) in the present study.

In the present study, mean hospital stay was found to be 9.6 days. 40 percent of the patients had hospital stay of less than or equal to 10 days. 3 patients each (30 percent of the patients each) had mean time to union of 12 weeks and 13 weeks respectively. Overall, mean time to union was found to be 12.45 weeks. Mean Harris hip score among patients on follow-up was found to be 92.5 . In 80 percent of the patients, mean Harris hip score was found to be 91 to 100 .

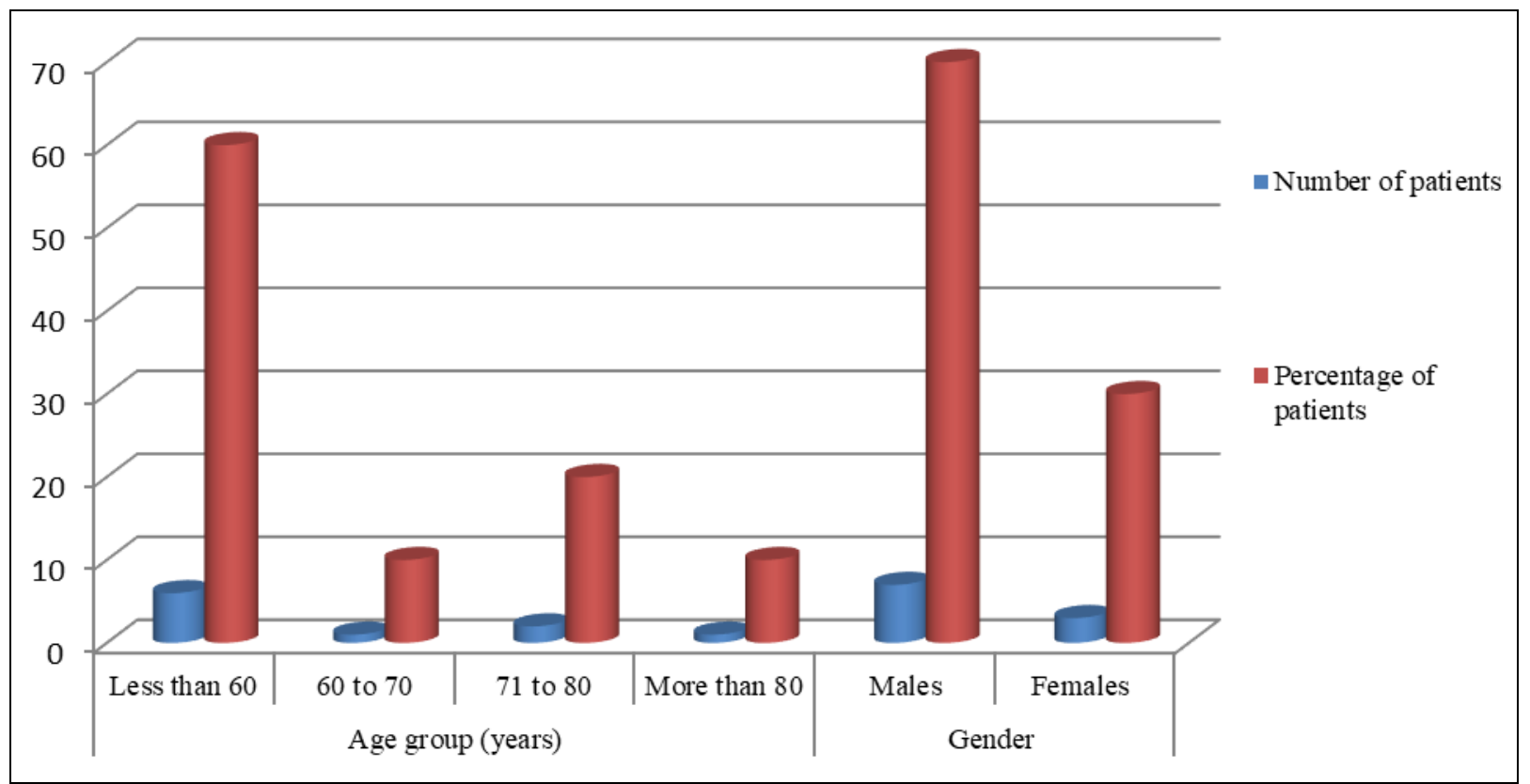

Graph 1: Age and Gender-wise distribution of patients

Table 1: Mean hospital stay

\begin{tabular}{|c|c|c|}
\hline Total hospital days & $\begin{array}{c}\text { Number of } \\
\text { patients }\end{array}$ & $\begin{array}{c}\text { Percentage of } \\
\text { patients }\end{array}$ \\
\hline Less than or equal to 10 & 4 & 40 \\
\hline 11 to 15 & 2 & 20 \\
\hline 16 to 20 & 3 & 30 \\
\hline More than 20 & 1 & 10 \\
\hline Total & 10 & 100 \\
\hline Mean & \multicolumn{2}{|c|}{$9.6 \pm 4.5$} \\
\hline
\end{tabular}

Table 2: Mean time to union (weeks)

\begin{tabular}{|c|c|c|}
\hline $\begin{array}{c}\text { Mean time to union } \\
\text { (weeks) }\end{array}$ & Number of patients & Percentage of patients \\
\hline 10 & 1 & 10 \\
\hline 11 & 2 & 20 \\
\hline 12 & 3 & 30 \\
\hline 13 & 3 & 30 \\
\hline 14 & 1 & 10 \\
\hline Total & 10 & 100 \\
\hline Mean & \multicolumn{2}{|c|}{$12.45 \pm 3.7$} \\
\hline
\end{tabular}


Table 3: Distribution of patients according to Harris hip score

\begin{tabular}{|c|c|c|}
\hline Total Score & Number of patients & Percentage of patients \\
\hline $71-80$ & 0 & 0 \\
\hline $81-90$ & 2 & 20 \\
\hline $91-100$ & 8 & 80 \\
\hline Total & 10 & 100 \\
\hline
\end{tabular}

\section{Discussion}

Apart from the general advantages of all cephalomedullary nails, the PFNA offers lesser trauma during insertion, lesser fluoroscopic and surgical times, lesser duration of hospitalization and a lesser rate of post-operative failure ${ }^{[8]}$.

In the present study, we analysed a total of 10 patients with inter-trochanteric femoral fractures who underwent treatment with PFNA. 60 percent of the patients of the present study were less than 60 years of age while 20 percent of the patients belonged to 71 to 80 years of age. Mean age of the patients of the present study was 58.8 years. There were 7 males (70 percent of the patients) and 3 females (30 percent of the patients) in the present study. Takigami et al. analyzed the results of treatment of trochanteric fractures in 50 patients with the PFNA. The average age of their patients was 84 years and majority of the fractures $(50 \%)$ were A1 type. They found that in four patients, the helical blade had slided laterally more than $10 \mathrm{~mm}$. However, as these patients were asymptomatic, this did not require re-operation. They encountered cut-out of the helical blade with non-union in one patient who eventually needed a bipolar hip prosthesis. They concluded that careful insertion of the helical blade or a sliding screw is a more important consideration that biomechanical stability for success of treatment using cephalomedullary devices. As compared to studies on the PFN, they found a reduced operative time and reduced blood loss. They regarded the PFNA as effective as other existing cephalomedullary devices in the treatment of trochanteric fractures [9]. Sahin et al. evaluated the functional and radiological outcome in 45 patients with unstable fractures which were treated using the PFNA. Radiological outcome was measured using the tip apex distance and the Cleveland and Bosworth system to assess position of the helical blade in the head/neck fragment. They achieved a TAD of less than $25 \mathrm{~mm}$ in $80 \%$ and an appropriate position of helical blade in $84.4 \%$ patients. After a mean follow up period of 17 months they achieved an average Harris Hip Score of 77.8. The scores were excellent in $24.4 \%$ patients and good in $42 \%$ patients. They encountered one case of cut out of the helical blade and asymptomatic lateral migration of the blade in four patients [10].

In the present study, mean hospital stay was found to be 9.6 days. 40 percent of the patients had hospital stay of less than or equal to 10 days. 3 patients each (30 percent of the patients each) had mean time to union of 12 weeks and 13 weeks respectively. Overall, mean time to union was found to be 12.45 weeks. Mean Harris hip score among patients on follow-up was found to be 92.5 . In 80 percent of the patients, mean Harris hip score was found to be 91 to 100. Gavaskar et al. in 2012 used the PFNA in 122 patients with an average age of 74 years with both stable and unstable fractures, the majority being A2 fractures. They included only low velocity falls in their study, essentially including only an osteoporotic set of patients. Union was achieved in all but one patient. Satisfactory reduction was obtained in 94 patients; all the unsatisfactory reductions occurred in unstable fracture types. They found that with unstable fractures, despite the nail theoretically being anti-varus collapse and anti-rotation, varus collapse was the most frequent complication accounting for two-thirds of all complications. They concluded that good results with relatively low complication rates can be achieved with the PFNA ${ }^{[11]}$. Sadic et al. in 2014 treated 63 patients with an average age of 73 years with the PFNA and sought to elucidate the effect of co-morbidities in the elderly to the return of function after surgery. They used the American Society of Anaesthesiologists (ASA) grading system to grade co-morbidities in their series. They found no statistically significant difference between different ASA grades with regard to post-operative complications or return of function. Although they did not use an objective scoring system to assess post-operative function, 38 of their 63 patients did not return to pre injury functional status ${ }^{[12]}$.

\section{Conclusion}

Under the light of above obtained results, it can be concluded that PFNA is accompanied by early recovery and appears to be an excellent mode of surgical therapy in patients with inter-trochanteric fractures.

\section{References}

1. Wolfgang GL, Bryant MH, O'Neill JP. Treatment of Intertrochanteric Fractures of the Femur Using Sliding Screw Plate Fixation. Clin Ortho. 1982; 163:148-58.

2. Pires R, Junior E, Santos L, Giordano V et al. Failure of fixation of trochanteric femur fractures: Clinical recommendations for avoiding $\mathrm{Z}$ effect and reverse $\mathrm{Z}$ effect type complications. Patient safety in Surgery. 2011; 5:17.

3. Strauss EJ, Kummer FJ, Koval KJ, Egol AJ. The "Zeffect" phenomenon defined: a laboratory study. J Orthop Res. 2007; 25:1568-1573.

4. Cheema G, Rastogi A, Singh V, Goel S. Comparison of cut out resistance of dynamic condylar screw and proximal femoral nail in reverse oblique trochanteric fractures: a biomechanical study. Indian Journal of Orthopaedics. 2012; 46(3):259-265.

5. Singh M, Nagrath AR, Maini PS. Changes in trabecular pattern in the upper end of the femur as an index of osteoporosis. J Bone Joint Surg Am. 1970; 52(1):457-67.

6. Pramudito J, Soegijoko $\mathrm{S}$ et al. Trabecular pattern analysis of proximal femur radiographs for osteoporosis detection. J Biomed Pharm Eng. 2007; 1(1):45-51.

7. Koot V, Kesselaer S et al. Evaluation of the Singh index for measuring osteoporsis. J Bone Joint Surg Br. 1996; 78(5):831.

8. Parker MJ, Handoll HH. Osteotomy, compression, and other modifications of surgical techniques for internal fixation of extracapsular hip fractures. Cochrane Database Syst Rev. 2009; (2):CD000522.

9. Takigami I, Matsumoto K, Ohara A, Yamanaka K. Treatment of trochanteric fractures with the PFNA (Proximal Femoral Nail Antirotation) nail system - report of early results. Bulletin of the NYU Hospitals for Joint diseases. 2008; 66(4):276-9.

10. Sahin S, Erturer E, Ozturk I, Toker S et al. Radiographic and functional results of osteosynthesis using the 
proximal femoral nail antirotation (PFNA) in the treatment of unstable intertrochanteric femoral fractures. Acta Orthop Traumatol Turc. 2010; 44(2):127-34.

11. Gavaskar A, Subramanian M, Tummala N. Results of proximal femoral nail antirotation for low velocity trochanteric fractures in the elderly. Indian Journal of Orthopaedics. 2012; 46(5):556-60.

12. Sadic S, Custovic S, Jasarevic M, Fazlic $\mathrm{M}$ et al. Proximal Femoral Nail Antirotation in treatment of Fractures of Proximal Femur. Med Arch. 2014; 68(3):173-77. 